REVISTA DE PSIQUIATRIA INFANTO-JUVENIL ISSN 1130-9512

Volúmen 36, número 3, julio-septiembre de 2019, p. 3-5

Este artículo está bajo licencia Creative Commons

BY-NC-ND 4.0

DOI: $10.31766 /$ revpsij.v36.n3a1

\section{Josefina Castro-Fornieles}

Servicio de Psiquiatría Infanto-Juvenil, 2017SGR881, Instituto de Neurociencias, Hospital Clinic de Barcelona, CIBERSAM, Universitat de

Barcelona

direccion.revista@aepnya.org

\section{Editorial}

\author{
Importancia de la formación en \\ psiquiatría de la infancia y \\ adolescencia
}

La adecuada atención a los trastornos mentales está siendo reconocida como prioritaria por parte de organismos y asociaciones internacionales debido a la alta prevalencia de estas patologías y su elevada repercusión para el funcionamiento y calidad de vida de los pacientes. Además, representa una gran carga social y económica $(1,2)$. Los recursos que se dedican por parte de los sistemas de salud para la prevención y tratamiento no son en general acordes con la valoración de dichas repercusiones (3).

La atención a la salud mental en las primeras etapas de la vida está empezando a ser prioritaria en salud pública (4) ya que en la infancia y adolescencia se inician muchos de estos trastornos $(5,6)$. Los años de vida ajustados por discapacidad perdidos por adolescentes y jóvenes relacionados estos trastornos son muchos (7). Además, la atención precoz a los problemas de salud mental puede prevenir consecuencias a largo plazo como por ejemplo en casos de inicio de consumo substancias o trastornos de conducta $(8,9)$. Los programas preventivos, que inicialmente pueden resultar costosos, han demostrado que comportan posteriormente una reducción del coste global del tratamiento, de la dependencia y de las consecuencias para la adaptación social de los pacientes $(10,11)$. Todo ello remarca la importancia de la atención adecuada a edades tempranas. Sin embargo, todavía existe una gran distancia en muchos países entre las necesidades reales de atención en salud mental en la infancia y adolescencia y la disponibilidad de recursos asistenciales (12).

La conceptualización de la psiquiatría de la infancia y la adolescencia durante los últimos sesenta años ha variado de forma importante. Las primeras clasificaciones de la OMS (13) contaban con muy pocos diagnósticos definidos como trastornos mentales infantiles. Las primeras asociaciones de psiquiatría de la infancia y la adolescencia no se crearon hasta los años 50. Por otro lado, se contaba con pocos tratamientos con suficiente base científica demostrada. En las últimas décadas se ha avanzado en la definición y criterios diagnósticos de numerosos trastornos que pueden presentarse en niños y adolescentes $(14,15)$, se ha dado valor al diagnóstico diferencial y se ha ido consolidando un modelo en el que se considera importante no solo la influencia del entorno si no también, las propias características del niño, ya sean genéticas, temperamentales o de personalidad. Todas estas influencias se consideran fundamentales no solo en la asistencia clínica sino también en los estudios de investigación (16).

La especificidad de la atención clínica en la infancia y adolescencia está avalada por muchos factores. A estas edades existen notables diferencias con los adultos en las manifestaciones de los trastornos ya que el cerebro está en desarrollo. Por otro lado, existe una gran influencia del entorno y de la familia en el estado del niño o adolescente y, además, los límites entre lo normal y lo patológico también están determinados por la etapa de desarrollo. Por todo ello, se necesitan centros clínicos experimentados en estas edades, que cuenten con profesionales formados en la utilización de diferentes fuentes de información y en instrumentos apropiados. También se precisa que en dichos centros se puedan realizar tratamientos específicos y diferenciados para los trastornos, tanto en abordaje psicoterápico como farmacológico, que tengan en cuenta las diferentes etapas evolutivas. 
En estas edades resulta aún más necesario abordar los trastornos de modo interdisciplinario y con la participación de diversos profesionales como psiquiatras, psicólogos, pediatras, enfermería o trabajo social, además de colaborar con maestros y psicólogos escolares. Es fundamental la implicación de la familia en el tratamiento del paciente para lograr mejores resultados. En algunos casos, es asimismo importante la interacción con las instituciones responsables de la atención a la infancia y la adolescencia (servicios de protección de menores, fiscalía y juzgados de menores). Toda esta coordinación implica un mayor tiempo de dedicación de los profesionales. El desarrollo de recursos asistenciales para salud mental en la infancia y adolescencia en muchos países ha sido menor en comparación con la atención a pacientes adultos, lo que dificulta el acceso al tratamiento específico o que en muchos casos la atención no se realice en el lugar más adecuado (12).

El reconocimiento de la importancia de la investigación en salud mental en edades tempranas también se ha ido incrementando en las últimas décadas ya que permite realizar estudios sobre diferentes trastornos sin la influencia de años de evolución ni de sucesivos tratamientos. Otro beneficio es que aporta conocimiento sobre los primeros síntomas y ello puede ser fundamental para la prevención. Aunque hay grandes diferencias entre los diversos países, en conjunto se puede decir que todavía la investigación en estas edades está en una situación por debajo de psiquiatría de adultos y de otras especialidades y, además, existe una gran distancia entre la clínica habitual y la investigación $(12,17)$. Evidentemente, el objetivo final de la investigación en salud mental de la infancia y adolescencia es mejorar la práctica clínica y se precisa incrementar la relación entre estas dos perspectivas tanto para optimizar la práctica cotidiana como para priorizar una investigación que conteste preguntas relevantes para el diagnóstico y los tratamientos (18). Por ello, se debe potenciar la investigación que incorpore diferentes disciplinas tanto básicas como clínicas. Algunos de los estudios en relación con la salud mental de la infancia y adolescencia que deberían ser promocionados con ayudas de fondos públicos serían los de factores de riesgo, prevalencia y seguimiento longitudinal de los trastornos, así como ensayos clínicos de calidad y de análisis de coste-eficacia de los diferentes abordajes $(12,19)$. También las normativas respecto a consentimientos informados y otros aspectos éticos en la investigación en estas edades han de ser claros y los comités éticos y agencias reguladoras suficientemente expertos en las especificidades para ayudar a la adecuada realización de los estudios.

De todo lo dicho anteriormente se deriva la importancia de contar con una formación en la especialidad de psiquiatría de la infancia y adolescencia regulada y que se realice adecuadamente y bajo un buen control de calidad. Es importante conseguir una formación homogénea y completa. Si no es así, es difícil que la atención a la salud mental en la infancia y adolescencia tenga la calidad necesaria, que los tratamientos sean homogéneos entre diferentes profesionales y que se sigan de forma correcta las guías internacionales. Por otro lado, si la formación en las primeras edades no es óptima se tendrán menos en cuenta sus especificidades y habrá dificultades en la detección y atención de muchos trastornos. En la mayoría de países europeos se reconoce la psiquiatría de la infancia y adolescencia como una especialidad diferenciada de la de adultos y se requiere una formación específica (17). En el estado español no está desarrollada la formación de esta especialidad y únicamente es obligatoria una formación de cuatro meses durante la residencia de psiquiatría. Una mayoría de residentes de psiquiatría desearían una mejor formación en esta área, pero actualmente suele ser escasa y muy variable. Se deja en gran parte a la libre elección del residente en función también de las posibilidades de su entorno (20). Es por tanto una necesidad que se apruebe oficialmente por las autoridades competentes la especialidad de psiquiatría de la infancia y adolescencia y que se regule y organice la formación de forma homogénea como sucede en los países del entorno. Otros beneficios que pueden obtenerse de una buena formación son una mayor identidad profesional ya que sus profesionales habrán decidido dedicarse a esta especialidad de forma voluntaria, priorizarán su red asistencial propia y pertenecerán a asociaciones específicas, potenciando así el conocimiento en esta área (21). Asimismo, es importante que los profesionales colaboren en disminuir el estigma y en mejorar la percepción pública de la atención a la salud mental infantil, así como de la investigación.

\section{REFERENCIAS}

1. Wittchen HU, Jacobi F. Size and burden of mental disorders in Europe-a critical review and appraisal of 27 studies. Eur Neuropsychopharmacol, 2005; 15:357-376.

2. Collins PY, Patel V, Joestl SS, March D, Insel TR, Daar AS et al. Grand challenges in global mental health. Nature 2011; 475:27-30. https://dx.doi.org/10.1038\%2F475027a

3. Vigo DV, Kestel D, Pendakur K, Thornicroft G, Atun R. Disease burden and government spending on mental, neurological, and substance use disorders, and self-harm: cross-sectional, ecological study of health system response in the Americas. Lancet Public Health, 2019 Feb; 4(2): e89-e96. https://doi. org/10.1016/S2468-2667(18)30203-2.

4. Patel V, Flisher AJ, Hetrick S, McGorry P. Mental health of young people: a global public-health challenge. Lancet 2007; 369: 1302-1313. https://doi.org/10.1016/S0140-6736(07)60368-7 
5. Gustavsson A, Svensson M, Jacobi F, Allgulander C, Alonso J, Beghi E, et al., Cost of disorders of the brain in Europe 2010. Eur Neruopsychopharmacol. 2011; 21:718-779. https://doi. org/10.1016/j.euroneuro.2011.08.008

6. Catalá-López F, Gènova-Maleras R, Álvarez-Martín E, Fernández de Larrea-Baz N, Morant-Ginestar C. Carga de enfermedad en adolescentes y jóvenes en España. Rev Psiquiatr Salud Ment. 2013; 6:80-85. https://doi.org/10.1016/j.rpsm.2012.07.002

7. Clavenna A, Cartabia M, Sequi M, Costantino MA, Bortolotti, Fortino I, et al. Burden of psychiatric disorders in the pediatric population. Eur Neuropsychopharmacol. 2013; 23: 98-106. https://doi.org/10.1016/j.euroneuro.2012.04.008

8. Newton-Howes G, Cook S, Martin G, Foulds JA, Boden JM. Comparison of age of first drink and age of first intoxication as predictors of substance use and mental health problems in adulthood. Drug Alcohol Depend. 2019; 194:238-243. https:// doi.org/10.1016/j.drugalcdep.2018.10.012

9. Kassing F, Godwin J, Lochman JE, Coie JD, Conduct Problems Prevention Research Group. Using early childhood behavior problems to predict adult convictions. J Abnorm Child Psychol. 2019; 47:765-778.

10. Motiwala SS, Gupta S, Lilly MB, Ungar WJ, Coyte PC. The cost-effectiveness of expanding intensive behavioural intervention to all autistic children in Ontario: in the past year, several court cases have been brought against provincial governments to increase funding for Intensive Behavioural Intervention (IBI). This economic evaluation examines the costs and consequences of expanding an IBI program. Healthc Policy. 2006; 1: 135-151

11. Bavarian N, Duncan R, Lewis KM, Miao A, Washburn IJ. Adolescent substance use following participation in a universal drug prevention program: examining relationships with program recall and baseline status. Subst Abus. 2015; 36:359-367. https:// doi.org/10.1080/08897077.2014.952364

12. Kieling C, Baker-Henningham H, Belfer M, Conti G, Ertem $\mathrm{I}$, Omigbodun $\mathrm{O}$, et al. Child and adolescent mental health worldwide: evidence for action. Lancet. 2011; 378:1515-1525. https://doi.org/10.1016/S0140-6736(11)60827-1

13. World Health Organization. Manual of the international statistical classification of diseases, injuries and causes of death. World Health Organization, 1957

14. World Health Organization. International Classification of Diseases 11th evision. World Health Organization, 2018

15. American Psychiatric Association. Diagnostic and statistical manual of mental disorders 5th edition (DSM-V), American Psychiatric Press, Washington, DC, 2013

16. M. Rutter. Routes from research to clinical practice in child psychiatry: restrospect and prospect. J Child Psychol Psychiatry. 1998; 39: 805-816.

17. Karabeiliroglu K, Dogangün B, Hergünter S, von Salis T, Rohtenberger A. Child and adolescent psychiatry training in Europe: differences and challenges in harmonization. Eur Child Adolesc Psychiatry. 2006; 15:467-475.

18. Palinkas LA, Um MY, Jeong CH, Chor KH, Olin S, Horwitz $\mathrm{SM}$, Hoagwood KE. Adoption of innovative and evidencebased practices for children and adolescents in state-supported mental health clinics: a qualitative study. Health Res Policy Syst. 2017; 15:27. https://dx.doi.org/10.1186\%2Fs12961-0170190-z

19. Hoagwood K, Olin SS. The NIMH blueprint for change report: research priorities in child and adolescent mental health. J Am Acad Child Adolesc Psychiatry. 2002; 41:760-767. https://doi. org/10.1097/00004583-200207000-00006

20. Fabrega M, Ilzarbe D. Becoming a child and adolescent psychiatrist in Spain: trainees' perspectives. Eur Child Adolesc Psychiatry. 2017; 26:743-745. https://doi.org/10.1007/ s00787-017-0974-2

21. McCarthy M, Abenojar J, Anders TF. Child and adolescent psychiatry for the future: challenges and opportunities. Psychiatr Clin North Am. 2009; 32: 213-226. https://doi. org/10.1016/j.psc.2008.11.008 